\title{
INFLUENZA IMMUNISATION RATES IN ADULTS, NSW 1993
}

Rob Menzies, AIDS / Infectious Diseases Branch Shing Chung Fung, Epidemiology Branch

NSW Health Department

his article provides a preliminary report of influenza immunisation rates among adults, based on data from the NSW Health Promotion Survey, 1994 (HPS) ${ }^{1}$.

Influenza prevention depends on the yearly immunisation of people at risk of serious complications following infection. During major epidemics hospitalisation rates may increase between twofold and fivefold ${ }^{2}$. It is estimated that more than 20,000 influenza-associated deaths occurred in each of 10 US epidemics between 1972 and $1990^{2}$. A recent meta-analysis concluded that influenza immunisation of elderly people reduced respiratory illness by 56 per cent, pneumonia by 53 per cent, hospitalisation by 50 per cent and deaths by 68 per cent ${ }^{3}$. Influenza immunisation has been shown to be a more cost-effective intervention than treatment of hypertension in middle-aged men, oestrogen therapy in postmenopausal women, neonatal intensive care and hospital haemodialysis ${ }^{4}$, and probably among the most cost-effective medical interventions in the older adult population ${ }^{5}$.

The National Health and Medical Research Council has recommended annual influenza immunisation for people at high risk of serious complications. At the time of the HPS, these groups were ${ }^{6}$ :

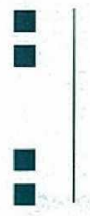
all people over 65 years of age; people of any age with chronic debilitating disease; especially cardiac, pulmonary, renal and metabolic disorders (including asthma and diabetes); people receiving immunosuppressive therapy; and health care personnel if particularly at risk.

Immunisation was not recommended for people outside these groups, as serious complications are unlikely and infection is regarded as providing longer-lasting immunity for a wider range of antigenic types of influenza.

\section{MetHODS}

The 1994 HPS was a telephone survey. It collected demographic information and data on a range of key health areas including injury, nutrition, sexual health, smoking and adult immunisation status. The study population included people 18 years of age or older in a household with a telephone number listed in the White Pages telephone directory (an estimated 93 per cent of NSW households have listed telephone numbers). One thousand interviews were conducted in each of 16 Health Areas or former Regions. All data presented here are estimates for the whole NSW adult population and have been produced by weighting for age, sex and geographic distribution using 1991 census data ${ }^{1}$.

Respondents were asked whether they had been immunised for influenza the previous year (1993). Data collected on risk categories for influenza complications were limited to age and past diagnosis of asthma or diabetes. Information on other chronic debilitating diseases and immunosuppressive therapy was not collected.

\section{RESULTS}

Sixteen thousand interviews were conducted. The response rate for those approached to be interviewed was 73 per cent. The overall estimate of the 1993 immunisation rate for the adult population was 13.5 per cent (99 per cent CI 12.8-14.2), corresponding to about 570,000 doses of vaccine. Among those 65 or more years of age (16 per cent of the adult population), 46.5 per cent (44.2-48.8) reported being immunised. Of those aged 18-64 years with asthma and/or diabetes ( 10 per cent of the population) 14.7 per cent (12.5-17.0) reported being immunised. Of the remaining people without recorded risk factors ( 73 per cent of the population), 6.0 per cent (5.4-6.6) reported being immunised.

At least 67 per cent of the doses of vaccine administered were given to people in high risk groups. Of those immunised, 59 per cent reported they had requested the

\section{Health of the people of NSW}

Continued from page 47

and/or long-term condition. In 1992, 52 per cent of deaths among older people were from cardiovascular diseases.

In 1992, 24 per cent of deaths among older people were from cancer. The most common sites for new cases of cancer in people aged 60 years and over were prostate, lung and colon cancer in men, and breast, colon and lung cancer in women.

In 1989-90, 89 per cent of older people reported an illness in the previous two weeks. The most commonly reported conditions were hypertension ( 34 per cent of older people) and arthritis (19 per cent). Ninety-four per cent of older people reported long-term conditions. Disorders of eyesight were reported by 62 per cent of older people and arthritis was reported by 37 per cent.

In 1993, 43 per cent of people aged 60-74 years had a disability, and 82 per cent of these had a handicap.
In those aged 75 years and over, 65 per cent had a disability, and 90 per cent of these had a handicap.

The report was compiled by the following present and former staff of the NSW Health Department's Public Health Division (in alphabetical order): Guncha Ansari, Gaston Arnolda, John Brown, Lucy Burns, Magnolia Cardona, Jennifer Chipps, Tim Churches, Glenn Close, Paul Corben, Stephen Corbett, Christine Cowie, Shing

Chung Fung, Margaret Kelaher, Ed Kraa, Michael Levy, Cait Lonie, Wendy Manning, Rob Menzies, Helen Moore, Geoff Morgan,

Ru Nguyen, Shanti Raman, Geoff Richards, Geoff Sayer, Gavin Stewart, Lyn Stoker, Lee Taylor and Margaret Williamson. The preparation of the report relied heavily on the Health Outcomes Information and Statistical Toolkit (HOIST), which was developed and is managed by Tim Churches and Peter Brandon.

Copies of the report are available from: The Better Health Centre, 162 Blues Point Road, North Sydney NSW 2060, Australia.

Phone: (02) 9954 1193; facsimile (02) 99555196.

The report can be accessed via the Internet from the NSW Health

Department's World-Wide Web site, at

http://www.health.nsw.gov.au/public-health/index.html 


\section{INFLUENZA IMMUNISATION RATES, 1993 BY RISK GROUP}

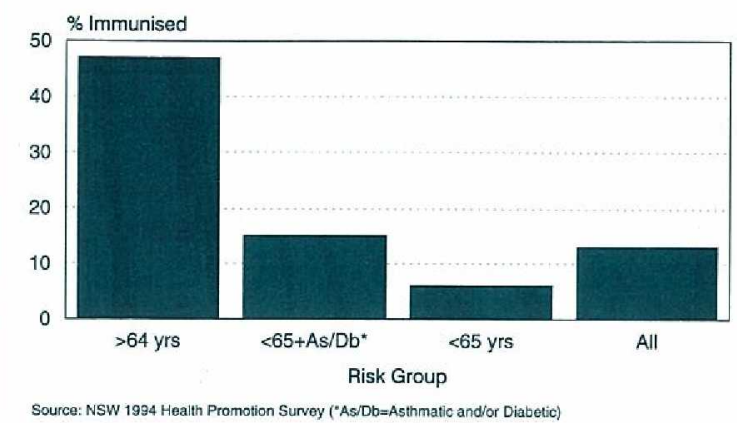

immunisation, while 36 per cent reported that it was recommended by their doctor.

\section{DISCUSSION}

The estimated immunisation rate ( 46.5 per cent) for people 65 years or over is similar to previous estimates of 45 per cent in Victoria in $1992^{7}$ and 52 per cent in South Western

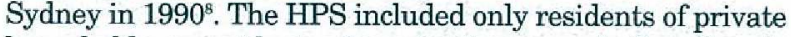
households, so residents of nursing homes and other chronic care facilities were excluded. In Victoria in 1992, 52 per cent of nursing home residents were reported to be immunised ${ }^{7}$. The immunisation rate in NSW residents 18-65 years with asthma and/or diabetes ( 15 per cent) was lower than those with chronic debilitating diseases in Victoria in 1992 ( 30 per cent).

One limitation of the HPS in monitoring influenza rates is that information was not collected on all indications for immunisation. Therefore, the group classified as not being at high risk (aged under 65 years without asthma and/or diabetes) includes an unknown number of people for whom immunisation was recommended. In the Victorian study, which included all age groups, 30 per cent of the surveyed population had at least one indication for immunisation, while in this study 27 per cent were either over 65 years of age or suffering from asthma or diabetes.

The estimated total number of doses given in NSW to adults from the HPS $(570,000)$ is slightly higher than estimates provided by the vaccine suppliers $(535,000)^{9}$.

The HPS showed that less than 33 per cent of vaccine doses were administered to people with no known indications. The actual figure may be lower, because of unreported indications. This compares with 47 per cent in Victoria in 1992 , which was a relatively severe influenza season during which vaccine supplies ran out and a second batch was produced. These data suggest that increased immunisation of people in low risk groups in response to publicity about a severe influenza season may have contributed to the shortage of vaccine in 1992. This underlines the importance of effective targeting of people in high risk groups during autumn, before the peak influenza season.

The majority of immunised people in high risk groups reported that immunisation was initiated by them ( 59 per cent) rather than by their doctors ( 36 per cent). It has been demonstrated that, regardless of a person's attitude to immunisation, 70 per cent to 90 per cent of people will accept a health professional's recommendation to be immunised ${ }^{10}$. This highlights the potential benefits from active advocacy of immunisation by health professionals.

In October 1994, after the HPS was conducted, the NHMRC recommendations were revised to the following ${ }^{11}$ :

It is recommended that the following groups should receive immunisation routinely:

- all persons over 65 years of age,

- Aboriginal and Torres Strait Islander people over 50 years of age.

Immunisation should also be considered for:

- adults with chronic debilitating diseases;

- children with cyanotic congenital heart disease;

- adults and children receiving immuno-suppressive therapy;

- staff who care for immuno-compromised patients; and

- residents and staff of nursing homes and chronic care facilities.

The immunisation rates detected by the HPS indicate that considerable public health benefits are being forfeited every year by the failure to immunise people at risk of serious complications from influenza. More aggressive strategies are needed to improve rates. In particular, general practitioners should actively encourage people in high risk groups to be immunised.

1. NSW Health Department. NSW Health Promotion Survey 1994 Technical Report. State Health Publication No: (HP) 950145.

2. Prevention and control of influenza: Recommendations of the Advisory Committee on Immunisation Practices. MMWR 1996; 45:RR-5. 3. Gross PA, Hermogenes AW, Sacks HS et al. The efficacy of influenza vaccine in elderly persons. A meta-analysis and review of the literature. Ann Intern Med 1995; 123:518-527.

4. Evans DB, Hensley MJ, O'Conner SJ. Influenza vaccination in Australia: a review of the economic evidence for policy recommendations. Med J Aust 1988; 149:540-543.

5. Hampson A. Influenza - dealing with a continually emerging disease. Communicable Disease Intelligence 1995; 20(9):212-216

6. National Health and Medical Research Council. Immunisation Procedures. 4th ed. Canberra: AGPS 1991.

7. MacIntyre CR, Carnie JA, Plant AJ. Influenza vaccination in Victoria, 1992. Med J Aust 1993; 159:257-260.

8. Brown J, Stewart G, Harris M, Coghlan M. Keeping the elderly covered. NSW Public Health Bulletin 1991; 2(10):102.

9. Personal communication, CSL and Rhone-Poulenc Rorer. 10. Fedson D. Address to Influenza Awareness Week launch, Sydney 1993.

11. National Health and Medical Research Council. The Australian Immunisation Procedure Handbook. 5th ed. Canberra: AGPS 1994. 